\title{
Food allergy and atopic eczema
}

By R. StC. BARnetson, Department of Dermatology, The Royal Infirmary, Edinburgh, and T. G. MERRETT, RAST Allergy and Research Unit, Benenden, Kent

The subject of food allergy is still viewed with considerable scepticism by the medical profession. There are several reasons for this but probably the most important is that few physicians or ecologists have yet made a serious attempt to analyse the condition critically. Food allergy is discussed in a number of books mostly emanating from the USA, particularly those by Rowe (1931), Coca (1942), Rinkel et al. (195I) and Dickey (1976). Unfortunately, each author uses the term 'food allergy' to describe both immunological and non-immunological phenomena, whereas most physicians would now reserve the term for hypersensitivity reactions to foods, particularly those mediated by IgE.

Foods may cause clinical manifestations for three main reasons:

1. The patient is intolerant of foods containing substances which

(a) have a pharmacological action (e.g. caffeine, tyramine)

(b) release chemical mediators (e.g. histamine)

(c) are toxic (e.g. acetanelide in rape-seed oil)

(d) are irritant to the intestinal mucosa, especially if it is already diseased (e.g. highly spiced foods)

2. The patient has an enzyme deficiency in the intestine such as lactase deficiency, leading to diarrhoea on ingestion of lactose-containing foods

3. The patient has true allergy to a food which, in comparison with other categories of food intolerance, is a relatively rare event

Most patients reporting to gastrointestinal clinics with supposed food allergy have no evidence of food hypersensitivity: many are neurotics, so providing another cause for scepticism by the medical profession.

Most patients who have IgE-mediated food allergy are atopic (Lessof et al. 1980) and a majority of these suffer from atopic asthma or eczema or both. It is still a controversial issue whether there is a definitive causal relationship between food allergy and atopic eczema and, if so, what mechanisms are responsible for the eczema. Whereas respiratory allergy has the expected hallmarks of IgE-mediated hypersensitivity, this is not true of atopic eczema where histological examination of the skin reveals that the main changes are oedema of the epidermis and infiltration of the epidermis and subepidermal region by mononuclear leucocytes.

Nevertheless, patients with atopic eczema, who very often suffer from respiratory allergy (asthma, allergic rhinitis) as well, tend to have much higher serum IgE concentrations than those with respiratory allergy alone (Fig. I). It has 


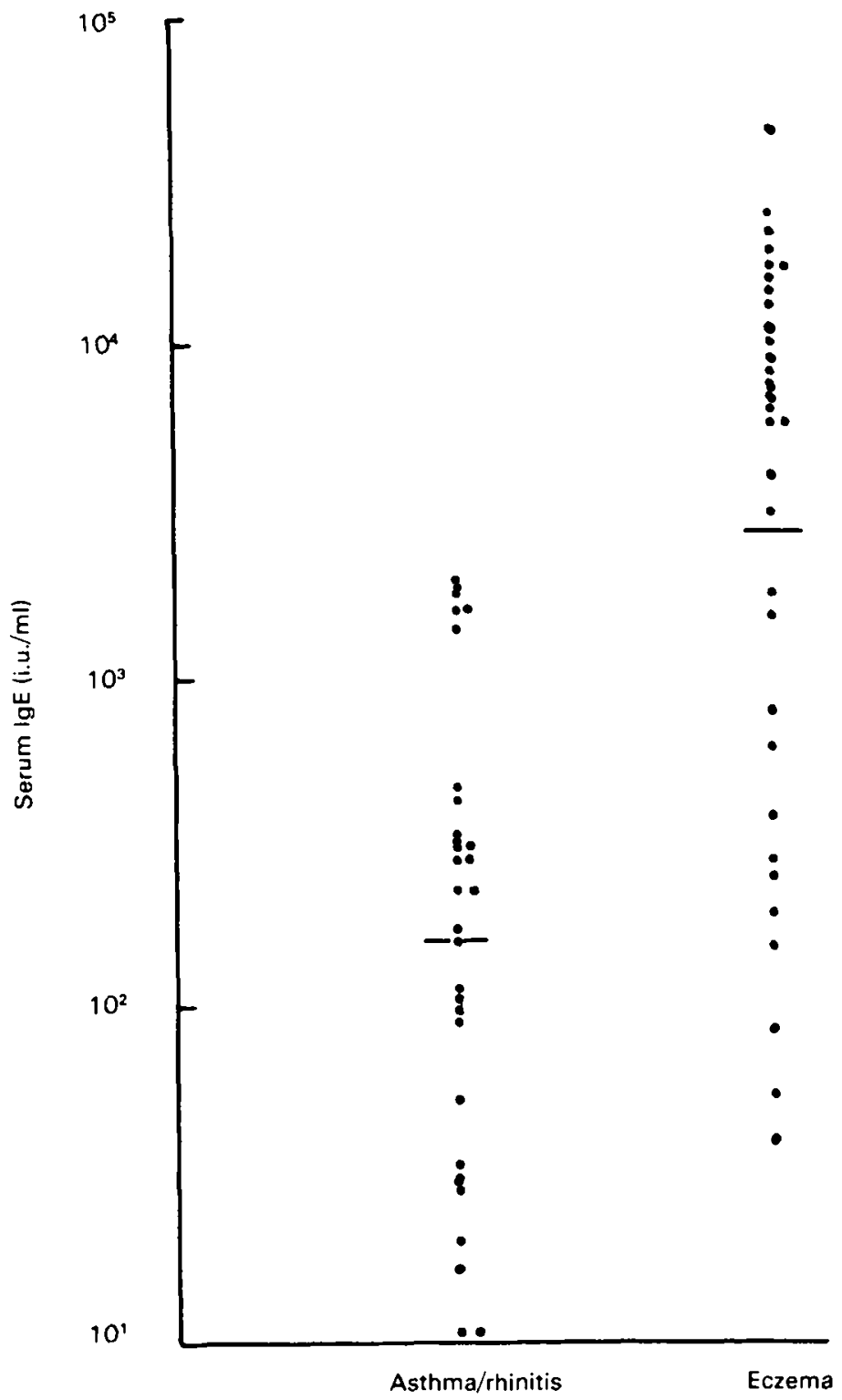

Fig. 1. Total serum IgE concentrations using a radio-immunosorbent test assay (Pharmacia; Uppsala, Sweden) in thirty-two patients with atopic eczema, twenty-five of whom had asthma or rhinitis, or both, compared with those in twenty-eight patients with asthma or rhinitis, or both, without eczema (upper limit of normal range $200 \mathrm{w} / \mathrm{ml}$ ). Medians are indicated by horizontal bars.

been shown (Barnetson, Merrett et al. 198I) that this results from two different factors: ( 1 ) a number of patients with eczema have a history of food allergy and 
several positive radio-allergo sorbent test (RAST) results to a panel of foods, and (2) patients in the eczema group tend to produce higher concentrations of specific IgE to inhalants than the respiratory allergy group (Fig. 2), presumably due to a lack of immunological suppression which may be a feature of atopic eczema (Strannegard, 1979; Leung et al. 1981).

If IgE antibodies to food allergens play a role in the pathogenesis of eczema, then the mechanisms involved are far from clear. It has been suggested that the skin

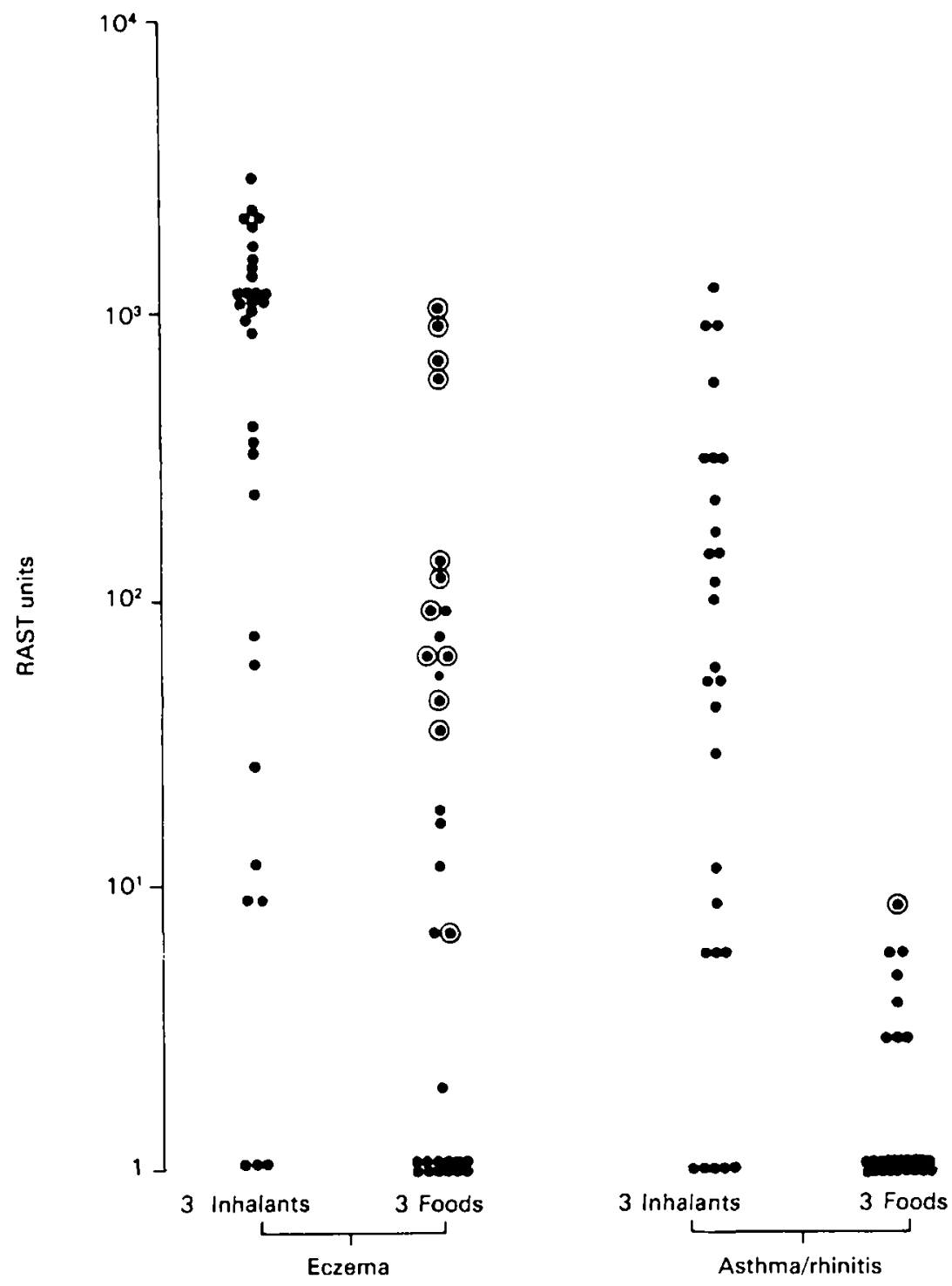

Fig. 2. The summed IgE antibody levels to three inhalants (grass pollen, house-dust mite, cat epithelium) and three foods (eggs, codfish, peanuts) in thirty-two eczema patients and twenty-eight patients with asthma or rhinitis, or both. Values for patients with clinical food allergy are circled. I RAST unit is equivalent to I i.u. total IgE (from Barnetson, Merrett et al. 198I). 
changes may result from the deposition of IgE complexes to food allergens absorbed from the gut (Brostoff et al. 1977) but this remains to be proved. Many patients with atopic eczema have neither a history of food allergy nor positive RAST and skin tests to foods: this suggests that IgE-mediated hypersensitivity to foods is the cause of atopic eczema in only a proportion of cases. However, Atherton et al. (1978), in a double-blind cross over trial, showed that exclusion of cows' milk and eggs from the diet of children improved eczema and that skin tests were unhelpful in predicting the efficacy of such a regimen. This might indicate that non-IgE-mediated mechanisms are involved in the pathogenesis of atopic eczema.

Patients with atopic eczema and evidence of food allergy have circulating, precipitating antibodies to foods in their serum (Barnetson et al. 1982). Atopic eczema patients also have high concentrations of subclass $\mathrm{IgG}_{4}$ in their serum (Shakib et al. 1977) and these are higher than in patients with respiratory allergy alone (Fig. 3). The role which $\operatorname{IgG}_{4}$ may play in allergy is currently being investigated in several laboratories. There are indications that it may act as a blocking antibody (Stanworth \& Smith, 1973) or conversely that it may be a second anaphylactic antibody responsible for the late reaction in asthma (Gwynn et al. 1982). However, others assert that $\mathrm{IgG}_{4}$ participates in neither role and may therefore just reflect immune-responsiveness. Eczema is usually not an immediate phenomenon and so we have determined the circulating levels of $\mathrm{IgG}_{4}$ antibodies to inhalant and food allergens in atopic eczema patients. Preliminary results show that these individuals produce more $\mathrm{IgG}_{4}$ to foods such as milk and eggs than to the common inhalants but of course these may not react with the same antigens as do IgE antibodies in the same sera.

The role of cell-mediated immunity to allergens such as foods in atopic eczema is also open to question. Allergic contact eczema has been shown to be a type of delayed hypersensitivity reaction where the allergen, usually a hapten such as nickel or chrome, comes into contact with the skin. The antigen-presenting cells in the epidermis are the Langerhans cells. It thus seemed possible that food allergens might cause a delayed hypersensitivity reaction in the skin following absorption of foods to which the patient had IgE-mediated hypersensitivity. However, it has been shown that patients with egg and milk allergies do not have high lymphocyte transformation responses to these allergens (Ferguson et al. 1983), and so there is as yet no evidence for delayed hypersensitivity reactions to foods playing any part in the pathogenesis of atopic eczema.

\section{Clinical manifestations of atopic eczema}

Atopic eczema usually presents in infancy; it often starts on the face and then spreads to the limbs involving the cubital and popliteal fossae, the ventral aspects of the wrists and the anterior aspects of the ankles. There is often a strong family history of atopy. Various precipitating factors have been suggested, such as cessation of breast-feeding, introduction of foods to the diet, infections, inoculations and teething. The role of breast-feeding in the pathogenesis is still to 


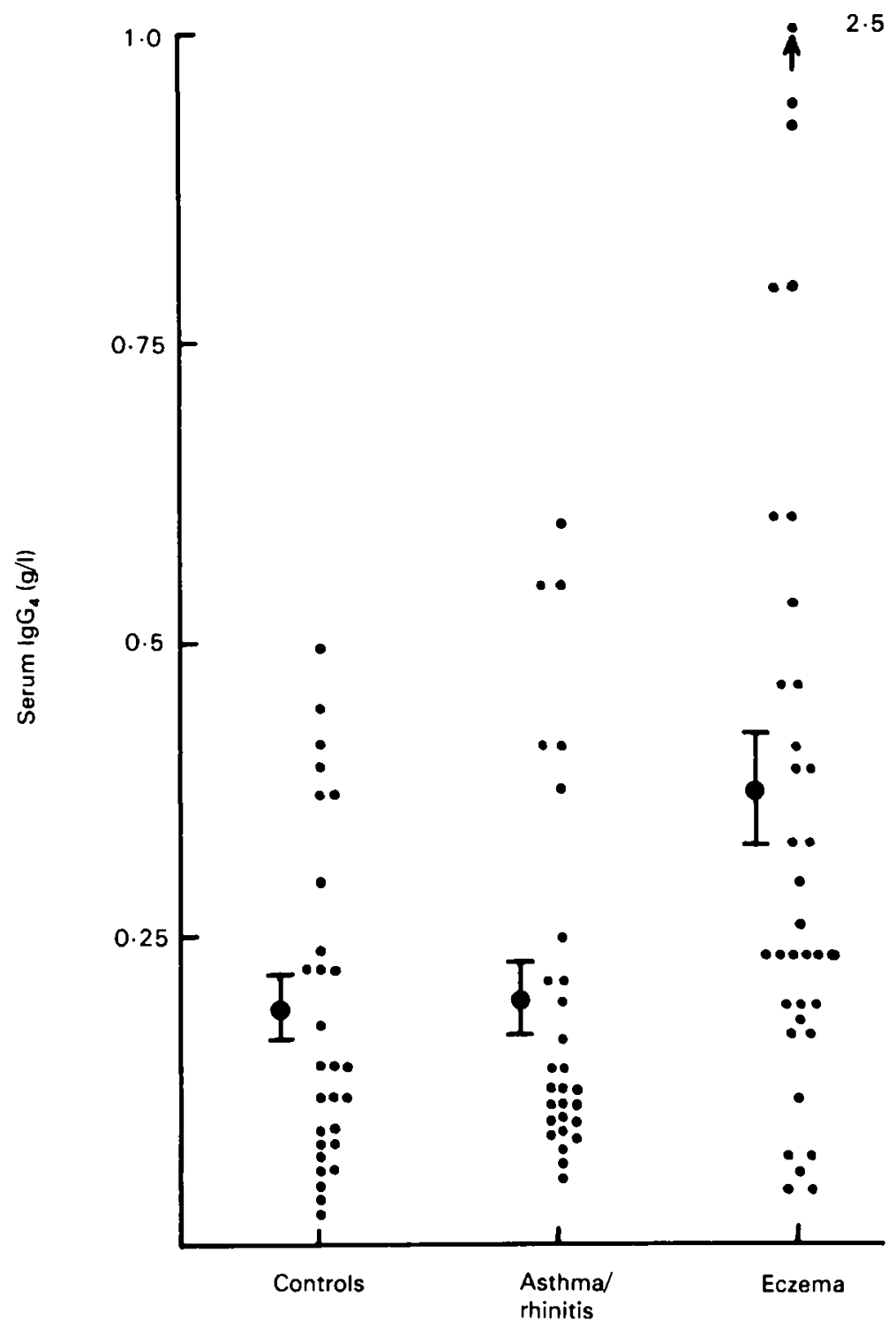

Fig. 3. Total serum $\mathrm{IgG}_{4}$ concentrations using a radio-immunodiffusion assay in thirty-five patients with atopic eczema compared with twenty-eight patients with asthma or rhinitis, or both, and twenty-eight age-matched healthy controls. Mean values are given, with their standard errors represented by vertical bars, for each group. Eczema patients compared with asthma and/or rhinitis and control groups: $P<0.0$ I for both, using the Student's $t$ test.

be settled. It seems likely that IgA in maternal mills plays an important role in the infant's immune response by guarding the respiratory and intestinal mucosa against infective agents and protecting the individual against the development of 
allergy to potential inhaled or ingested antigens. Taylor et al. (1973) have produced evidence that atopic children have a delayed production of IgA, which would make them more vulnerable to potential allergens, but this has yet to be confirmed by other workers.

Introduction of foods other than maternal milk is likely to be relevant, as most foods are potentially allergenic: those likely to be implicated are cows' milk, hens' eggs, wheat, fish, shellfish and nuts, though the first two allergens will usually be the most important in infancy, especially if the child is not breast-fed. Infections (Frick et al. 1979) and inoculations are also likely to play some part in the pathogenesis of atopy, as both may modulate immune regulation. It is interesting to note that Bordetella pertussis is used as an adjuvant to induce a high IgE response in experimental rats and it might possibly produce a similar response in humans, particularly if there is a familial history of atopy. The role of teething in eczema is hard to explain, though it may just be a stressful situation: stress is a common exacerbating factor in atopy. Patients with atopic eczema usually resolve spontaneously before puberty, possibly due to thymic maturation. However, if the eczema continues into adulthood and is widespread, it presents a serious problem.

These patients may well have other manifestations of atopy such as asthma which, like eczema, is often outgrown at puberty, and rhinitis which often starts in the early teens. A proportion also suffer from food allergy which may present as angio-oedema and urticaria following ingestion of the food, vomiting of the food or refusal and dislike of certain foods. The latter is a common story, and a history of food refusal may indicate food allergy in some children which can be confirmed by skin testing and RASTs. As is the case in eczema and asthma, patients often outgrow their food allergy, and this is particularly true of allergy to cows' millk. In contrast, food allergy may also be acquired and thus may occur during infections such as infectious mononucleosis (Barnetson, Hardie et al. I98I) where there is likely to be modulation of immune regulation.

\section{Investigation of food allergy in atopic eczema}

It is important to stress that not all patients with atopic eczema have food allergy: some do not have positive skin tests to any common allergen. It might be argued that such patients are not atopic by definition, but if they present the characteristic clinical picture and they have a family history of atopy then the diagnosis is fairly certain. It is possible that they have outgrown any allergies and thus have negative skin tests. However, if food allergy exacerbates the patient's eczema, then it is important to be aware of the fact. Often, patients with food allergy and atopic eczema have severe eczema which will not be controlled by simple measures such as the topical application of steroids.

Total serum $\operatorname{IgE}$. This is a useful assay in the assessment of older children and adults. A vast majority of patients with atopic eczema and food allergy have very high concentrations and if the serum IgE is within normal limits $(<200 \mathrm{u} / \mathrm{ml})$ then food allergy is unlikely. The most useful test for assaying $\operatorname{IgE}$ in atopic eczema patients is the radio-immunosorbent test (RIST) (Pharmacia; Uppsala, Sweden) 
where patients tend to have high IgE concentrations. However, the most accurate method is the radio-immune assay described by Merrett \& Pantin (1975).

Skin ('prick') tests to food allergens. These tests in our opinion are much more useful in the diagnosis of IgE-mediated food allergy than the literature would suggest. They are cheap and easy to perform, and give an 'instant' result. They can be performed on children of any age, and the procedure is virtually painless when performed carefully. Previous problems with the skin test have resulted mainly from impurity of allergens used in the test, and from the frequency of false positives. The latter problem results in part from the fact that manufacturers of skin test allergens have suggested that any wheal greater in diameter than the control indicates food allergy. Patients with atopic eczema frequently produce multiple positive skin tests, using such criteria, probably as a result of their high total serum IgE concentrations. If a skin test is only regarded as positive when a wheal of more than $5 \mathrm{~mm}$ in diameter is produced, then the risk of false positives will be reduced to a minimum and a better correlation with history of food allergy and positive RASTs obtained.

Radio-allergo sorbent tests to foods in atopic eczema patients. The RAST is a useful way of assaying specific IgE allergens but at present there are considerable disadvantages in using such an assay. Besides the problems of cross reactivity, and the fact that a number of plants act as lectins, the solid phase has an important non-specific IgE-trapping effect (Johansson \& Bjorksten, 1980), particularly in those who have high concentrations of $\operatorname{IgE}$ in their serum, i.e. most atopic eczema patients.

In the most frequently used assays for specific IgE in Europe (Pharmacia; Uppsala, Sweden) a score system of $0_{-4}$ is suggested, where 0 is negative, $I$ is doubtful positive and 2, 3 and 4 are increasingly positive. Our experience shows that, generally speaking, only RAST scores of 3 or 4 correlate with the history of food allergy and with positive skin tests. Many atopic eczema patients produce scores of 2 to multiple food allergens: this is probably due to non-specific binding to the discs (see Table I). One other problem is the fact that a RAST score of 4 may represent a specific IgE concentration of $17.5 \mathrm{u} / \mathrm{ml}$ or more. As assays of serum from some of our patients have shown that they produce up to $5000 \mathrm{u}$ $\mathrm{IgE} / \mathrm{ml}$ to one allergen, most commonly to inhalants such as house-dust mite, this means that the present score system is totally inadequate for research studies, particularly those performed on a sequential basis.

Provocation tests in atopic eczema. These must be performed on a double-blind basis and should be repeated three times. They may be useful in those patients who assert that they or their children develop severe eczema after ingestion of a food, though their skin tests and RASTs to such a food are negative.

\section{Treatment of food allergy in the amelioration of atopic eczema}

It should be stressed once again that not all atopic eczema is related to food allergy. It is obviously useful to perform skin tests and RASTs to food allergens to identify foods which may cause IgE-mediated hypersensitivity. However, other 


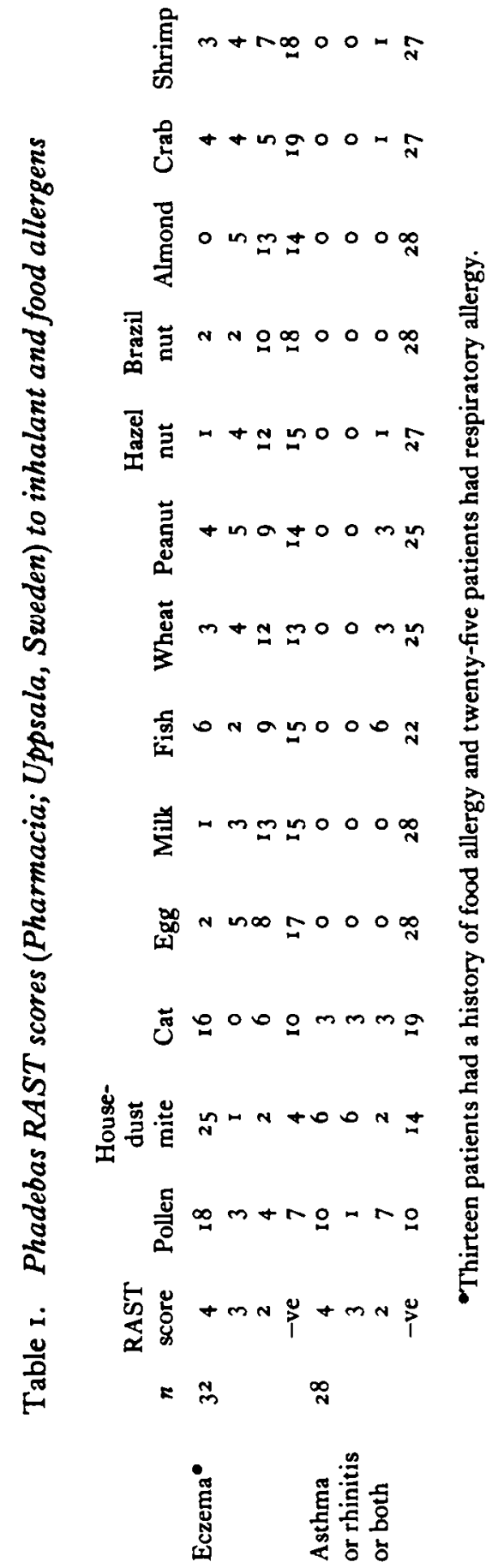


types of hypersensitivity may be involved, and the role of precipitating antibodies to foods and of $\mathrm{IgG}_{4}$ in the pathogenesis of eczema remains to be elucidated. If hypersensitivity to foods is suspected to be an important precipitant of a patient's eczema, there are three useful approaches to the problem.

I. The patient is started on an elemental diet, such as Vivonex (Norwich Eaton; Woking), for 2 weeks to see if their eczema clears. Foods are then introduced, a group at a time, over a period of 6 months, provided there has been a good response to the elemental diet. This is extremely time-consuming and does not take into account the fact that other precipitating factors such as stress may cause an exacerbation which is attributed wrongly to a food.

2. Diet exclusion is useful in some patients. In our experience, those with allergy to fish can avoid ingestion or any contact with fish, and thus perform their own exclusion diet. However, those who are allergic to eggs and milk, which are common constituents of cooked foods, may do well by starting on a diet which is completely egg-free or milk-free, and a number of patients have had their eczema cleared by such an approach.

3. The use of anti-allergic drugs such as disodium cromoglycate. The case for the use of this drug in patients with atopic eczema remains to be proved. It is likely to be effective only in those patients with allergy to common foods, such as eggs and cows' milk, but may be preferable to exclusion diets which limit the patient's food choice very considerably.

\section{REFERENCES}

Atherton, D. J., Sewell, M., Soothill, J. F., Wells, R. S. \& Chilters, C. E. D. (1978). Lancet i, 401. Barnetson, R. StC., Ferguson, A. \& Drummond, H. (1982). Br. J. Derm. ro7, Suppl. 22, 15. Bametson, R. StC., Hardie, R. A. \& Merrett, T. G. (1981). Br. med. F. 283, 1086. Barnetson, R. StC., Merrett, T. G. \& Ferguson, A. (1981). Clin. exp. Immunol. 46, 54 Brostoff, J., Johns, P. \& Stanworth, D. R. (1977). Lancet ii, 74I.

Coca, A. F. (1942). Familial Non-reaginic Food Allergy. Springfield, Illinois: Charles C. Thomas. Dickey, L. D. (I976). Clinical Ecology. Springfield, Illinois: Charles C. Thomas.

Ferguson, A., Strobel, S. \& Barnetson, R. StC. (1983). Medicine et Hygiene. (In the Press.)

Frick, O. L., German, D. F. \& Mills, J. (1979). F. Allergy clin. Immunol. 63, 228.

Gwynn, C. M., Ingram, J., Almousawi, T. \& Stanworth, D. R. (I982). Lancet i, 254.

Johansson, S. G. O. \& Bjorksten, F. (1980). Allergy 35, 177.

Lessof, M. H., Wraith, D. G., Merrett, T. G., Merrett, J. \& Buisseret, P. D. (1980). Quart. F. Med. $195,259$.

Leung, D. Y. M., Rhodes, A. R. \& Geha, R. S. (1981). Y. Allergy clin. Immunol. 67, 450.

Merrett, T. G. \& Pantin, C. F. A. (1975). Clin. Chim. Acta 65, 131.

Rinkel, H. J., Randolph, T. G. Y. \& Zeller, M. (1951). Food Allergy. Springfield, Illinois: Charles C. Thomas.

Rowe, A. H. (1931). Food Allergy, its Manifestations and Diagnosis and Treatment with a General Discussion of Bronchial Asthma. Philadelphia: Lee \& Febiger.

Shakib, F., McLaughlan, P., Stanworth, D. R., Smith, E. \& Fairbairn, E. (1977). Br. f. Derm. 97, 59 . 
Stanworth, D. R. \& Smith, A. K. (1973). Clin. Allergy 3, 37.

Strannegard, I. L. (1979). Int. Arch. Allergy Appl. Immunol. 58, i 75.

Taylor, B., Norman, A. P., Orgel, H. A., Stokes, C. R., Turner, M. W. \& Soothill, J. F. (I973). Lancet ii, 111 . 